\title{
Motion control of a compliant wheel-leg robot for rough terrain crossing
}

\author{
Arthur Bouton $^{a, b}$, Christophe Grand ${ }^{a, c}$ and Faïz Benamar ${ }^{a, b}$
}

\begin{abstract}
In this paper, we propose the use of compliant elements in the actuation of a wheel-legged robot in order to improve its locomotion properties on unknown and irregular terrains. Detection of the obstacles is achieved by a synergistic use of the structural compliances. The robot's capabilities to surmount steep obstacles is thus improved thanks to the inertia of the chassis and flexibility in postural control. In the proposed robot's kinematics, the four wheels are attached to the main body through vertical series elastic actuators (SEA) and with a passive horizontal compliant mechanism subject to a specific wheel speed control. The overall control relies on postural servoing and a local reactive loop which adapts the vertical forces applied by the SEA on each wheel according to the detected obstacle and the stability margin. The resulting system is evaluated with physical simulations for two case studies : a canonical steep obstacle on one wheel at a time and multiple random rough terrains.
\end{abstract}

\section{INTRODUCTION}

Hybrid locomotion systems using wheels at the end of actuated legs take advantage of their additional degrees of freedom to maximize stability [1], optimize ground force distribution [2] and to adopt special locomotion behaviors [3], [4], [5]. On such systems, the optimization of locomotion properties such as the force distribution needs very accurate placement of the wheels relative to the ground due to the system's indeterminacy, especially when the actuation stiffness is high. As the knowledge of the ground geometry remains a challenging issue, the wheel-legged robots need to progress very slowly just to maintain constant contact between wheels and ground.

On the other hand, wheeled robots using passive suspensions with large displacement, such ones encountered in planetary exploration [6], offer a native posture adjustment that improves the wheels' pressure distribution on soil [7] and helps to cross over obstacles with particular shape thanks to the suspension kinematics [8]. However, these systems are not able to react to some discontinuities in ground profile by for example adapting the wheel-soil contact forces. Furthermore, their stability remains dependent on the ground geometry.

In this work, we propose to combine the best of these two approaches by developing a robot that uses an active compliant locomotion system. The technological aspects are not taken into account for the moment, so we consider a purely theoretical model where the compliant elements are decomposed as seen on Fig. 1 . The frame $\mathcal{R}=(\vec{x}, \vec{y}, \vec{z})$ is

\footnotetext{
${ }^{a}$ Sorbonne Universités, UPMC Univ Paris 06, UMR 7222, ISIR, F-75005, Paris, France $\{$ bouton, amar\}@isir.upme.fr

${ }^{b}$ CNRS, UMR 7222, ISIR, F-75005, Paris, France

${ }^{c}$ ONERA - The French Aerospace Lab, Toulouse, France christophe.grandeonera.fr
}

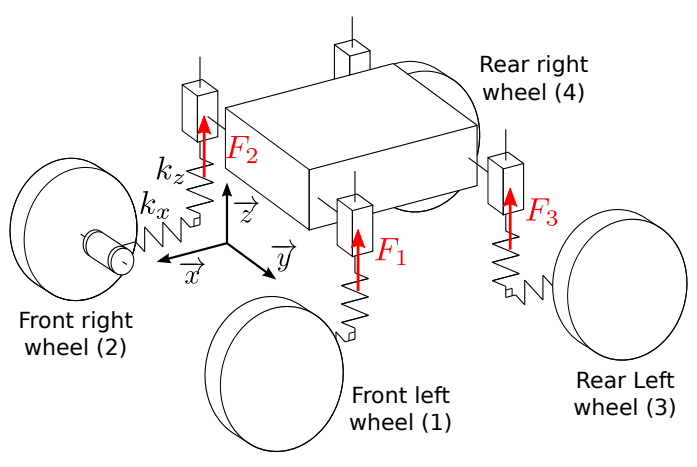

Fig. 1. Decomposition of compliances

attached to the robot chassis where $\vec{x}$ denotes the longitudinal axis and $\vec{z}$ the body vertical axis.

At each wheel, we consider two orthogonal compliance terms. The horizontal one, along $\vec{x}$ axis with a stiffness coefficient $k_{x}$, is passive and tends to keep constant space between the wheels. It plays a significant role in maintaining the adherence when approaching vertical obstacles [9]. The vertical compliance term $k_{z}$ is associated with a linear actuator to form a series elastic actuator as defined in [10]. Its principle is outlined on Fig. 2 : a desired input force is compared to the one applied by the elastic deformation and the force error is used to compute the position variation of the stiff linear actuator. While the spring is free to absorb ground irregularities at high frequency, this actuation principle allows control of the vertical force balance between each wheel regardless of the soil profile. The differences in height of the ground are then naturally filtered and the wheels tend to be maintained in contact thanks to a permanent residual positive force.

In this architecture, the vertical forces are controlled while the horizontal wheel positions are maintained at a reference by the springs and a specific wheel speed control : a decomposition that respects the force/position orthogonality principle [11]. The control of vertical forces has been previously suggested in [12], but here we don't consider neither the wheel traction, which can not practically be assessed, nor

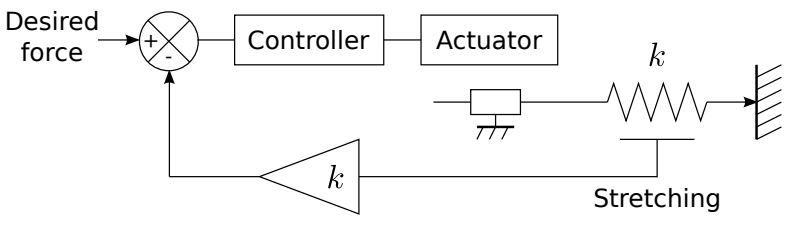

Fig. 2. Series elastic actuators principle 


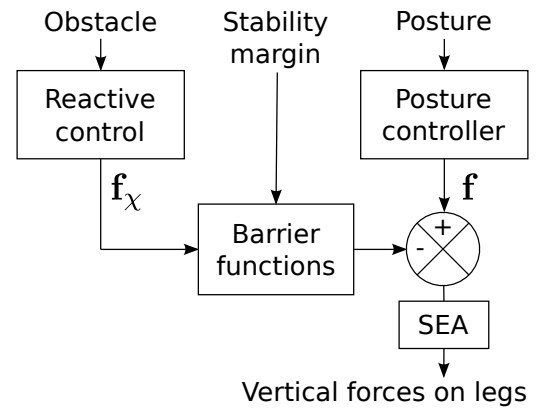

Fig. 3. Overview of the proposed control architecture

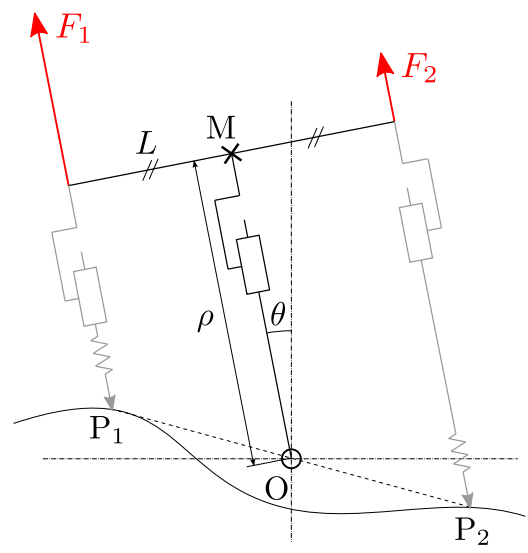

Fig. 4. Pendulum model for postural control

the quasi-static equilibrium from the contact forces. Instead, the main idea is to exploit the synergy of both transversal compliances in order first to reduce the number of actuators compared to wheel-leg systems, and secondly to improve the dynamic adaptation of the locomotion system to the terrain irregularities and especially to abrupt obstacles at higher speeds.

The organisation of the paper is led by the overall control architecture as depicted in Fig. 3 First, we describe the postural control that computes the vertical forces $f$ to be applied by the SEA actuators. After that, we introduce a criterion to detect and measure the surmounting difficulty of obstacles. Then, we describe the reactive controller that computes a correction term $\mathbf{f}_{\chi}$ subtracted to the forces $\mathbf{f}$ obtained by the posture controller. In order to ensure the tipover stability of the robot, we define barrier functions that moderate the value of $\mathbf{f}_{\chi}$ related to a stability margin. Last, the proposed system and its control are evaluated with simulations considering two case studies : a canonical steep obstacle and randomly generated rough terrains.

\section{POSTURAL CONTROL}

The posture adjustment aims at controlling both the center of mass (CoM) elevation and the main body pitch and roll angles. This is achieved by modeling the system as an inverted linear pendulum in which the rotational axis would be at the center of a segment joining the wheel-soil contact points. Fig. 4 gives a 2D representation of the model in the frontal plane.
The robot posture is controlled by the forces $F_{i}(i \in[1,4])$ applied by the SEA actuators at fixed lengths $L$ of the CoM M. Note that these forces can only be positive due to the unilaterality of the contacts, so a total external acceleration (mainly the gravity) oriented toward the ground is needed in order to keep the system controllability. This way, we can easily express the dynamical relation between the four vertical forces embedded in $\mathbf{f}=\left[\begin{array}{llll}F_{1} & F_{2} & F_{3} & F_{4}\end{array}\right]^{T}$ and the state vector $\mathbf{q}=\left[\begin{array}{lll}\rho & \theta_{x} & \theta_{y}\end{array}\right]^{T}$ :

$$
\mathbf{M}(\mathbf{q}) \ddot{\mathbf{q}}=\mathbf{G} \mathbf{f}+\mathbf{N}(\mathbf{q}, \dot{\mathbf{q}})+\eta,
$$

where $\mathbf{N}(\mathbf{q}, \dot{\mathbf{q}})$ contains gravity, centrifugal and Coriolis forces. $\theta_{x}$ and $\theta_{y}$ are the pitch and roll angles. $\rho$ is the CoM elevation, which is computed from the mean of legs vertical length. These correspond to the SEA prismatic joints parameter, noted $r^{i}(i \in[1,4]) . \eta$ is unknown perturbation forces that have to be rejected by the controller.

In a similar way of what it is done in humanoid balancing [13], the posture is adjusted thanks to a PD controller with a possible dynamical decoupling :

$$
\mathbf{f}=\mathbf{G}^{+}\left(\mathbf{M}(\mathbf{q})\left(\mathbf{K}_{\mathbf{p}} \widetilde{\mathbf{q}}+\mathbf{K}_{\mathbf{d}} \dot{\mathbf{q}}\right)-\mathbf{N}(\mathbf{q}, \dot{\mathbf{q}})\right),
$$

where $\widetilde{\mathbf{q}}$ is the difference between the actual and the reference posture and $\mathbf{G}^{+}$the pseudo-inverse of $\mathbf{G}$ which gives the vector $f$ with minimum robot's internal stresses. This vector provides the input forces for the SEA control. In order to maintain wheels in contact with the ground, we include a small positive lower bound on these forces.

The reference posture is here chosen with $\vec{z}$ aligned with the gravity so that the weight is equally distributed on each wheel for a centered CoM. Furthermore, it allows to keep the decomposition of compliances according to the support efforts and the progression ones without additional actuation. Finally, the elevation $\rho$ is chosen as function of the rover geometry to have sufficient ground clearance.

\section{WHEEL VELOCITY ADAPTATION}

Because of passive compliances, relative displacements in wheel horizontal positions entails persisting internal stresses maintained by ground friction. Furthermore, when wheels are on slopes of different angles, it's necessary to adapt their speed in order to give them the same horizontal velocity component without slippage. Therefore, we choose to modulate each wheel speed $\omega$ proportionally to their deviation according to the relation :

$$
\omega=\left(1+k_{\omega} l_{x}\right) \frac{V_{x}^{d}}{R},
$$

where $V_{x}^{d}$ is the desired longitudinal velocity of the whole robot, $R$ is the radius of the wheel and $l_{x}$ is the stretching of the horizontal spring from its nominal position. We define $l_{x}$ positive when the wheel is displaced towards the rear of the vehicle. $k_{\omega}$ is then a control coefficient that should be chosen large enough to minimize the settling time with respect to changes in slope, without generating instability in the horizontal chassis displacements when subject to speed variations. 


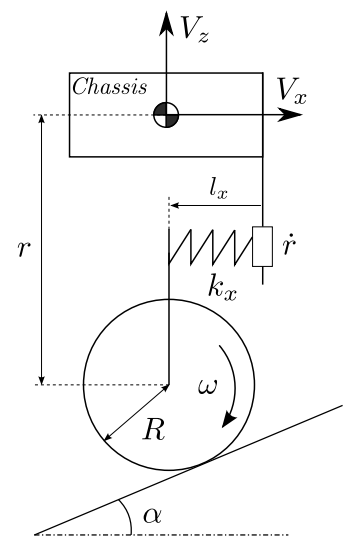

Fig. 5. Wheel kinematics on a slope

The wheel kinematics for a slope of angle $\alpha$ is shown in Fig. 5, where $V_{x}$ and $V_{z}$ are respectively the actual horizontal and vertical main body velocities. Let $\dot{r}$ be the vertical component of the wheel velocity with respect to the main body. Then, if there is no slippage and the system is at the kinematic balance $\left(\dot{l}_{x}=0\right)$, the relation from the decomposition of velocities gives :

$$
V_{x}^{2}+\left(\dot{r}+V_{z}\right)^{2}=(R \omega)^{2},
$$

which leads to :

$$
\frac{\dot{r}}{V_{x}^{d}}=\sqrt{\left(1+k_{\omega} l_{x}\right)^{2}-\left(\frac{V_{x}}{V_{x}^{d}}\right)^{2}}-\frac{V_{z}}{V_{x}^{d}} .
$$

Therefore, assuming that the actual horizontal speed $V_{x}$ of the main body is close enough to the desired one and that its vertical speed $V_{z}$ is negligible compared to $V_{x}$, the expected vertical velocity of the wheel $\dot{r}_{c}$ can be expressed as a function of the horizontal compliant element's deformation and the desired traveling velocity :

$$
\left\{\begin{array}{l}
V_{x} \approx V_{x}^{d} \\
V_{z} \ll V_{x}^{d}
\end{array} \Rightarrow \dot{r}_{c} \simeq V_{x}^{d} \sqrt{\left(1+k_{\omega} l_{x}\right)^{2}-1} .\right.
$$

\section{OBSTACLE CROSSING}

\section{A. Obstacle Detection}

Eq. (6) gives a theoretical value for the vertical displacement of legs when fulfilling the non slip condition. Then, this theoretical value $\dot{r}_{c}$ can be compared with the measured one $\dot{r}$, which depends on the vertical compliances. When the wheel have trouble overcoming an obstacle, $\dot{r}$ is smaller than the expected value $\dot{r}_{c}$. However, as both $\dot{r}_{c}$ and $\dot{r}$ fluctuate a lot on rough terrains, more restrictive conditions are needed in order to prevent misdiagnosis. First, $l_{x}$ should be positive, which ensures that the wheel may be pushed back by an obstacle. Then, a positive torque indicates that the wheel is indeed working positively to track the required speed. In practice, the sign of the torque can easily be obtained by the sign of the motor current. The last interferences from the relatives movements of legs can be avoided by applying the initial condition of detection $\dot{r}_{c}-\dot{r}<0$, while $l_{x}$ and $\tau$ are positive. This condition reflects the precedence of vertical bouncing with respect to the horizontal displacement of the wheel due to inertia when it reaches an obstacle.

Thus, we can build a function $\chi$ which will indicate the presence of an obstacle :

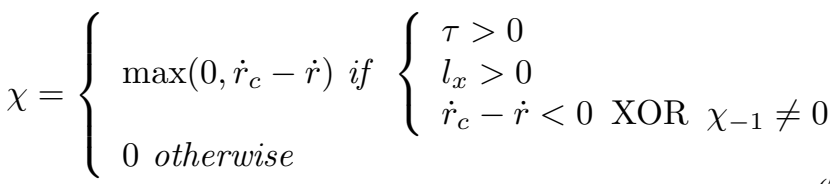

where $\tau$ is the torque of the wheel and $\chi_{-1}$ is the previous value of $\chi$. The function $\chi$ allows not only the detection of obstacles but also to quantify the difficulty to overcome them.

\section{B. Reactive Control}

Restraining the value of this function $\chi$ to zero helps to overcome steep obstacles. This can be achieved by acting on the wheel with a force proportional to the speed difference $\chi$, which allows a smooth reaction near zero, thereby preventing force oscillations in SEA. As we don't know the exact part of the wheel traction in the vertical forces, we have to gradually adapt the reactive control until it is enough to reach the theoretical vertical speed without causing the sign of the wheel torque to become negative. Hence we add an integral term, which leads to a PI controller on $\chi$ (cf. Fig. 6) for each leg. These controllers compute the vector of reactive forces $\mathbf{f}_{\chi}$ that are subtracted to the leg forces $\mathbf{f}$ from the postural control.

Such an acting on the wheels is enabled by the inertia of the chassis, whose fast movements entail forces on legs. So to prevent robot's tipover from an excessive modulation of the support forces, we propose to implement barrier functions to narrow the effect of the reactive loop according to a stability margin.

\section{Stability Safeguard}

The robot is subject to the gravity $\vec{g}$, and also accelerations $\overrightarrow{a_{e}}$ due to the variations of its motion in the inertial frame. They can come from ground undulations, steering dynamics, or speed variations. The last case happens whenever the robot comes up against an obstacle and entails large horizontal accelerations. Then, we have to ensure a sufficient margin in order to prevent the risks of tipover from a collision at any moment.

As shown on Fig. 7, tipover occurs as soon as the direction of $\vec{g}-\overrightarrow{a_{e}}$ is out of the support polygon defined by the contact points with the ground. In such a situation, the unilaterality of contacts does not allow the robot to counter the moment applied around the edge of the polygon. A margin known as the Force-Angle stability measure [14] has been defined and often used in previous works dealing with this risk. It consists in the product of the resultant force norm exerted on the robot excepted from the contacts, here equal to $\left\|M \vec{g}-M \overrightarrow{a_{e}}\right\|$, and the minimal angle between this resultant and the vector joining the CoM to the edge of the support polygon. It has been shown that this margin is the optimal one when considering inertial effects [15]. However, 


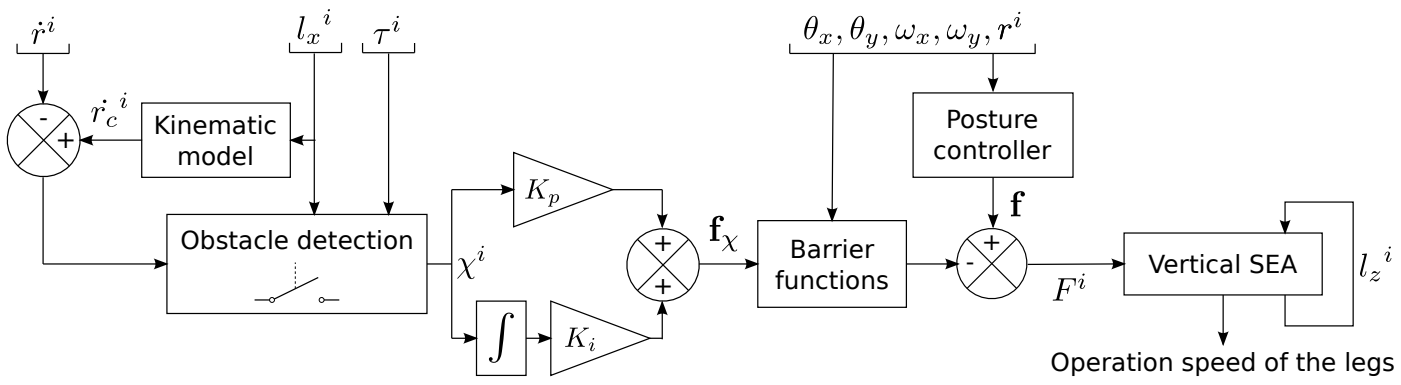

Fig. 6. Diagram of the control

due to the force control, we cannot precisely know the robot's acceleration without an accurate measurement of the angular accelerations of the chassis. But these accelerations have brief variations and our interest isn't to quantify the stability for any situation, but to know its boundaries. For this reason we solely consider the limit angle from the tipover possibility, which depends on the position of the CoM relative to the points of contact. Thus we introduce the angle $\gamma$ which refer to the maximum perturbation we want the robot to be able to overcome at any moment. This angle, chosen beforehand, can vary in time according to the speed of the robot or its turning rate for example. From the ground contacts point of view, this security margin draws a pyramidal space in which the center of mass has to stay, as depicted in Fig. 8 .

The equations of the four planes that define the pyramid can be expressed in the cartesian coordinate centered in $\mathrm{O}$ :

$$
\begin{aligned}
& -x-\tan (\gamma) z+d_{1}=x-\tan (\gamma) z+d_{2}=0 \\
& -y-\tan (\gamma) z+d_{3}=y-\tan (\gamma) z+d_{4}=0
\end{aligned}
$$

Thus, we can assess the distance $D^{i}(i \in[1,4])$ from the CoM position to these planes and apply a barrier function which is equal to 0 when the distance is null and tends to 1 when it's infinite. This function is of the form $D^{i} \mapsto e^{-\frac{\sigma}{D^{i}}}$, where $\sigma$ is a repulsive coefficient. The force $f_{\chi}$ obtained for each wheel from the reactive control is then multiplied by the barrier functions of both adjacent planes. While staying within the stability limits, the reactive control is free to modify the forces in the legs. Otherwise, the barrier functions give back the priority to the postural control.

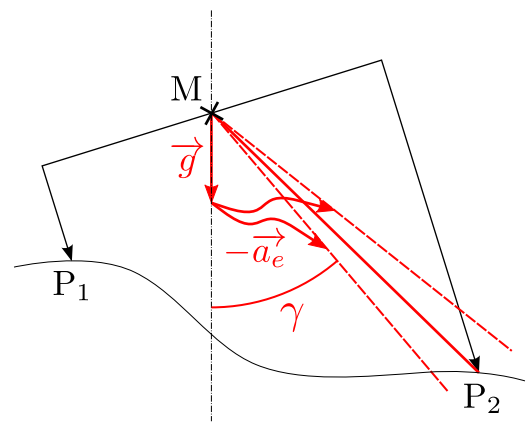

Fig. 7. Illustration of the dynamic stability margin

\section{SIMULATIONS RESULTS}

The physical simulations are based on Open Dynamics Engine (ODE). We use a pyramidal approximation for the friction model with a coefficient of 0.5 , which corresponds to a tire on a wet road. In order to simulate tires softness, contacts dynamics is configured in ODE through two parameters : a CFM (Constraint Force Mixing) coefficient of $0.02 \mathrm{~m} \mathrm{~s} \mathrm{~N}$ and a ERP (Error Reduction Parameter) coefficient of 0.5 The simulation step is set to $1 \mathrm{~ms}$.

The robot is modeled with a total weight of $M=12 \mathrm{~kg}$, a $0.6 \mathrm{~m}$ wheelbase, $0.15 \mathrm{~m}$ wheel radius and an horizontal stiffness of $k_{x}=1 \mathrm{kN} \mathrm{m}^{-1}$. The wheel speeds are modulated with $k_{\omega}=10 \mathrm{~m}^{-1}$. The gains of the reactive control have been tuned in order to properly cross the canonical obstacle described above, what led to $K_{p}=1 \mathrm{kN} \mathrm{m}^{-1} \mathrm{~s}$ and $K_{i}=$ $10 \mathrm{kN} \mathrm{m}^{-1}$. The stability margin $\gamma$ is fixed at $30^{\circ}$ and $\sigma$ at $0.01 \mathrm{~m}$.

\section{A. Analysis of a canonical obstacle crossing}

To visualize the effects of the reactive control, we use a canonical obstacle made by a unique vertical box whose height is $2 / 3$ of the wheel diameter and placed in front of both right wheels of the robot. The obstacle is chosen thin enough to let the front wheel reach the ground again before the rear wheel comes in contact with it because it represents the hardest case for the rear wheel approach according to the static equilibrium.

The robot reaches the obstacle with a speed of $0.6 \mathrm{~m} \mathrm{~s}^{-1}$. Fig. 9 gives an overview of the simulation while the robot

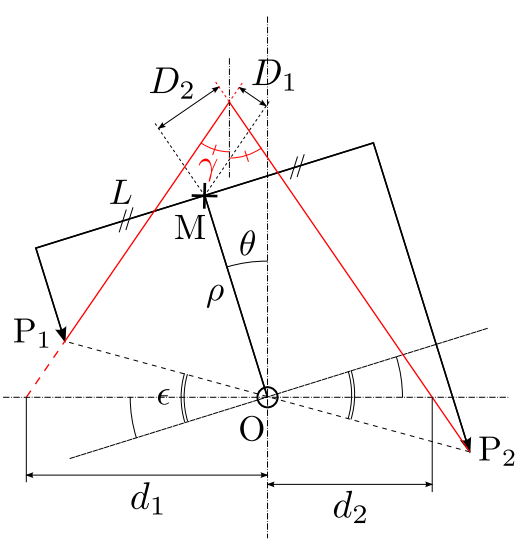

Fig. 8. Valuation of the stability margin 


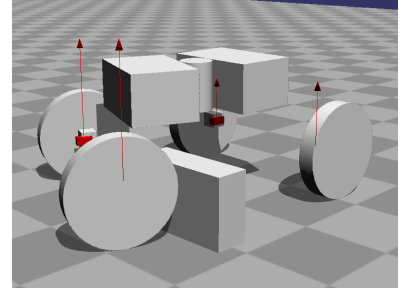

(a) Postural control only (b) Reactive control

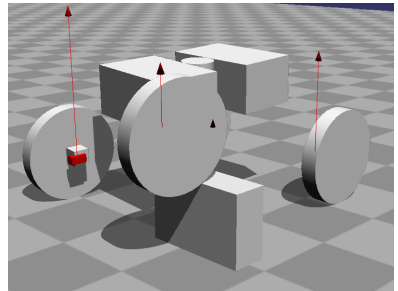

Fig. 9. Insight of the canonical obstacle crossing

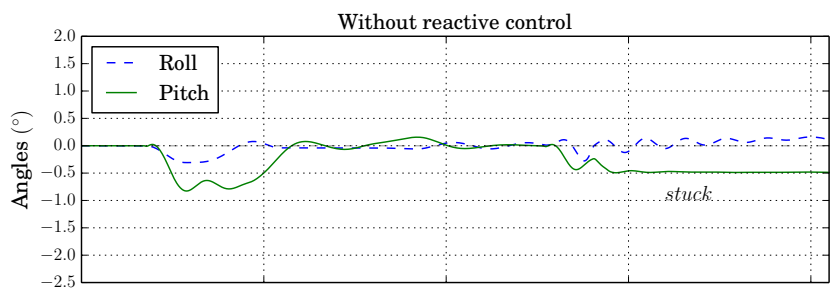

With reactive control

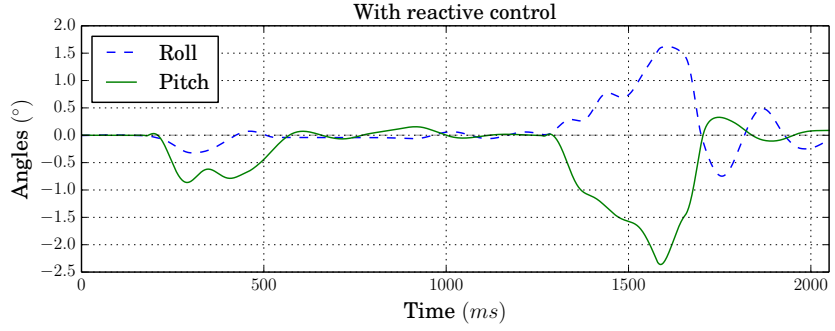

Fig. 10. Inclinations during the canonical obstacle crossing

is using the reactive control (vertical arrows represent the control forces) to overcome the obstacle.

With the postural control alone, the robot is not able to surpass because the static equilibrium does not allow its rear wheel to ride over the obstacle. On the other hand, with the reactive control the robot relaxes some of its stability margin at the right time to let the wheel step over the obstacle. This can be seen in Fig. 10 where the roll and pitch angles are compared for both cases. We notice at $200 \mathrm{~ms}$ that the equilibrium is identical since the front wheels go over obstacle in both case without difficulty. The detection function $\chi$ from Eq. (7) is then near zero and the reactive control is insignificant. Then, at $1250 \mathrm{~ms}$ we observe that when the rear wheel comes in contact with the obstacle, the reactive control induces a tilt on the robot. As soon as the wheel is out of difficulty, the robot recovers its initial balance.

Fig. 11, which lets us compare the obstacle detection function $\chi$ of all the wheels together, shows how the reactive control suppresses the surmounting difficulty.

The controller's impact can be observed on Fig. 12 since the measured vertical link speed of the wheel-leg $\dot{r}$ is forced to follow the theoretical one only when the wheel risks to be stuck (at $1250 \mathrm{~ms}$ ) thanks to the detection conditions from $\chi$. We can notice that the perturbations from the other wheel motions when the front right wheel climbs over the obstacle at $200 \mathrm{~ms}$ and descends at $900 \mathrm{~ms}$, are filtered. Finally, the reactive control stops when the right rear wheel has reached the top of the obstacle at $1600 \mathrm{~ms}$, although the horizontal displacement takes some time to be relaxed.

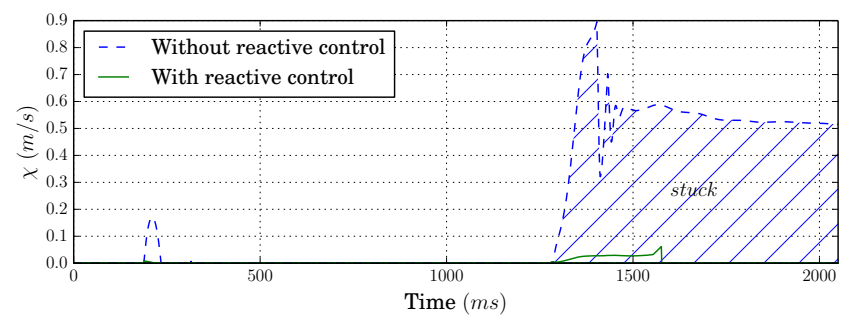

Fig. 11. Obstacle detection

\section{B. Performance comparison}

Fig. 13 shows the maximum height $H_{\max }$ the robot can cross according to its speed. The obstacle is the same one as described in the previous section but we change its height up to the overcoming limits for each speed. This height is shown as a percentage of the wheel diameter. Compared to passive suspensions we can then appreciate an improvement in performance with the use of SEA for the postural control, and even more with the reactive one.

Also, we then want to compare the robustness of the system over rough terrains with many obstacles as shown on Fig. 14. Thus we randomly generate numerous asymmetrical terrains consisting of two independent juxtaposed tracks (at the left side and the right side of the robot). On each one, the obstacles are randomly positioned according to a uniform law every $0.6 \mathrm{~m}$ step. Each element is also subject to a random rotation over $\vec{y}$, offering various orientations of planes for the wheels. We then judge that the robot succeed to cross the terrain if it reaches the end of the tracks ( $10 \mathrm{~m}$ further) and the wheels stay on their respective track of $0.6 \mathrm{~m}$ width. The obstacles' height follows a normal distribution with a standard deviation of $0.15 \mathrm{~m}$ and a mean that we vary. Fig. 14 depicts an example of terrain obtained for an obstacles' mean height of $0.3 \mathrm{~m}$. Fig. 15 reveals the success rates after one thousand trails over one thousand different random terrains for each size of obstacles. The three types of suspensions run successively over each generated terrains with a same speed of $1.2 \mathrm{~m} \mathrm{~s}^{-1}$. The passive suspensions used as a reference
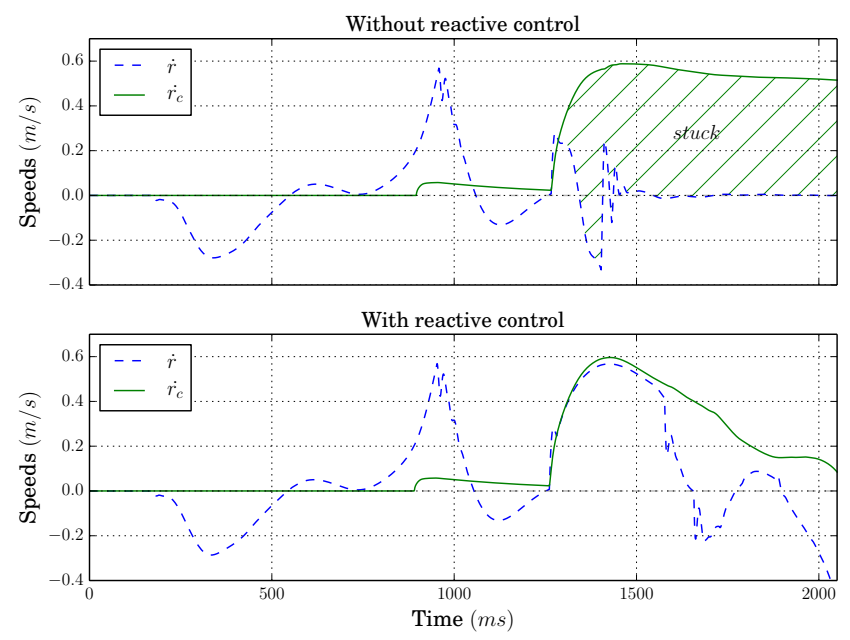

Fig. 12. Kinematically estimated and measured speeds of the rear right leg displacement 


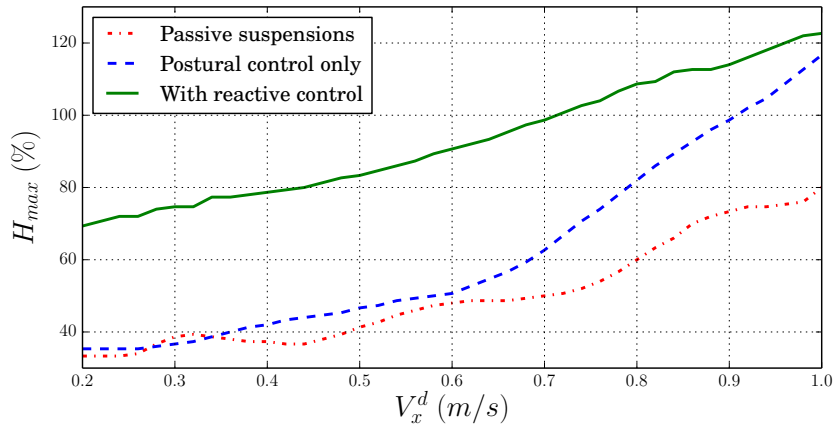

Fig. 13. Maximum crossable height on a vertical surface

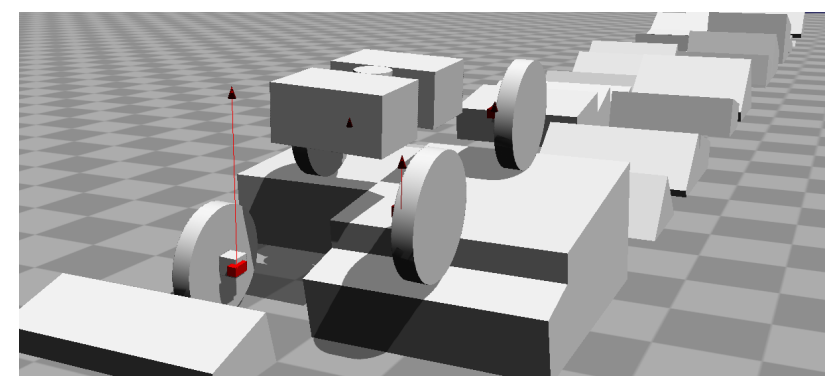

Fig. 14. Overview of the rough terrain

have been tuned to reach good performances over these terrains, which has led to a vertical stiffness of $600 \mathrm{~N} \mathrm{~m}^{-1}$ and a damping of $20 \mathrm{~N} \mathrm{~m}^{-1} \mathrm{~s}$, while the horizontal compliance is the same for each kind of suspension. In order to reach the target position, the robot is steered thanks to a central joint in the chassis. So we notice the significant efficiency of the force control in the legs to deal with the miscellaneous reliefs. Then, the reactive control gives an additional increase in the chances of success.

The video available at http: / /www. isir.upmc. fr/ vid/compliant_crossing.mp4 gives a visual comparison of the suspensions over a sample of terrains and for the case of a bilateral step obstacle.

\section{CONCLUSIONS}

A suitable decomposition of the compliances allows the robot to control its posture while ensuring continuous wheelsoil contact and obstacle detection. This detection is achieved

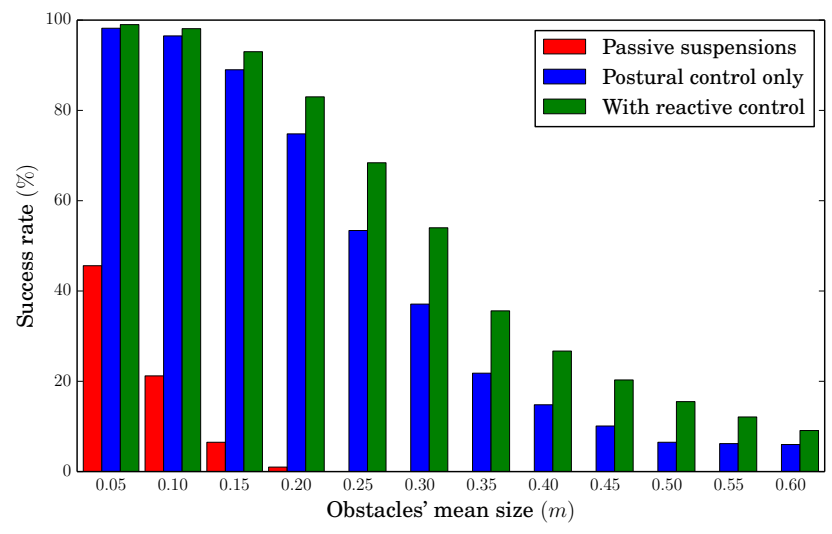

Fig. 15. Results on the rough terrain thanks to the synergy of vertical and horizontal compliances and by evaluating the kinematic constraint of the nonslippage condition that reveals abnormal vertical wheel-leg velocity.

The posture is controlled by means of series elastic actuators used to regulate the vertical component of the wheel contact forces and to balance the dynamic perturbations using an inverted pendulum model. A complementary controller, local to each wheel, uses a metric indicating the presence of an obstruction and modulates the contact force in order to help the wheel to overcome obstacles by exploiting the inertial forces of the chassis. The robot configuration is also evaluated during the motion and the effect of force modulations is limited according to the distance with a stability margin. This produces a novel kind of compliance resulting from flexibility in the posture.

With the whole control architecture, the robot moves over rough terrains without the need of prior knowledge on their geometry while ensuring a certain stability margin. This allows it to go faster and use more kinematic energy in order to help high obstacles crossing. In future works, we will consider the design of an experimental prototype and its evaluation on real situations.

\section{REFERENCES}

[1] J. A. Smith, I. Sharf, and M. Trentini, "Paw: a hybrid wheeled-leg robot." in Robotics and Automation (ICRA), IEEE Int. Conf. on, 2006.

[2] P. Jarrault, C. Grand, and P. Bidaud, "Robust obstacle crossing of a wheel-legged mobile robot using minimax force distribution and selfreconfiguration," in Intelligent Robots and Systems (IROS), IEEE/RSJ Int. Conf. on, 2011, pp. 2753-2758.

[3] A. Halme, I. Leppnen, M. Montonen, and S. Ylnen, "Robot motion by simultaneously wheel and leg propulsion," in Proc. CLAWAR, 2001.

[4] C. F. Steeves III, "Design and behavioural control of a dynamic quadruped with active wheels," Ph.D. dissertation, McGill University, 2002

[5] F. B. Amar, C. Grand, G. Besseron, and F. Plumet, "Performance evaluation of locomotion modes of an hybrid wheel-legged robot for self-adaptation to ground conditions," in Proc. of ESA Workshop on Advanced Space Technologies for Robotics and Automation, 2004.

[6] V. Kucherenko, A. Bogatchev, and M. Van Winnendael, "Chassis concepts for the exomars rover," in Proc. of ESA Workshop on Advanced Space Technologies for Robotics and Automation, 2004.

[7] T. Thueer, A. Krebs, R. Siegwart, and P. Lamon, "Performance comparison of rough-terrain robotssimulation and hardware," Journal of Field Robotics, vol. 24, no. 3, pp. 251-271, 2007.

[8] R. Siegwart, P. Lamon, T. Estier, M. Lauria, and R. Piguet, "Innovative design for wheeled locomotion in rough terrain," Robotics and Autonomous systems, vol. 40, no. 2, pp. 151-162, 2002.

[9] A. K. Singh, R. K. Namdev, V. Eathakota, and K. M. Krishna, "A novel compliant rover for rough terrain mobility," in Intelligent Robots and Systems (IROS), IEEE/RSJ Int. Conf. on, 2010, pp. 4788-4793.

[10] G. A. Pratt and M. M. Williamson, "Series elastic actuators," in Intelligent Robots and Systems (IROS), IEEE/RSJ Int. Conf. on, 1995.

[11] L. Villani and J. De Schutter, "Force control," in Springer Handbook of Robotics, 2008, pp. 161-185.

[12] V. P. Eathakota, A. K. Singh, and K. M. Krishna, "Two models of force actuator based active suspension mechanisms for mobility on uneven terrain," Acta Astronautica, vol. 67, no. 9, pp. 1233-1247, 2010.

[13] B. J. Stephens and C. G. Atkeson, "Dynamic balance force control for compliant humanoid robots," in Intelligent Robots and Systems (IROS), IEEE/RSJ Int. Conf. on, 2010, pp. 1248-1255.

[14] E. Papadopoulos and D. A. Rey, "A new measure of tipover stability margin for mobile manipulators," in Robotics and Automation (ICRA), IEEE Int. Conf. on, 1996, pp. 3111-3116.

[15] E. Garcia, J. Estremera, and P. G. De Santos, "A comparative study of stability margins for walking machines," Robotica, vol. 20, no. 06 , pp. 595-606, 2002. 